\title{
Theoretical analysis of BER performance of nonlinearly amplified FBMC/OQAM and OFDM signals
}

\author{
Hanen Bouhadda ${ }^{1} 2^{*}$, Hmaied Shaiek ${ }^{1}$, Daniel Roviras ${ }^{1}$, Rafik Zayani ${ }^{2}$, Yahia Medjahdi ${ }^{3}$ \\ and Ridha Bouallegue ${ }^{2}$
}

\begin{abstract}
In this paper, we introduce an analytical study of the impact of high-power amplifier (HPA) nonlinear distortion (NLD) on the bit error rate (BER) of multicarrier techniques. Two schemes of multicarrier modulations are considered in this work: the classical orthogonal frequency division multiplexing (OFDM) and the filter bank-based multicarrier using offset quadrature amplitude modulation (FBMC/OQAM), including different HPA models. According to Bussgang's theorem, the in-band NLD is modeled as a complex gain in addition to an independent noise term for a Gaussian input signal. The BER performance of OFDM and FBMC/OQAM modulations, transmitting over additive white Gaussian noise (AWGN) and Rayleigh fading channels, is theoretically investigated and compared to simulation results. For simple HPA models, such as the soft envelope limiter, it is easy to compute the BER theoretical expression. However, for other HPA models or for real measured HPA, BER derivation is generally intractable. In this paper, we propose a general method based on a polynomial fitting of the HPA characteristics and we give theoretical expressions for the BER for any HPA model.
\end{abstract}

Keywords: FBMC/OQAM; OFDM; HPA; Nonlinear distortion; BER

\section{Introduction}

Nowadays, 4G systems such as 3GPP-LTE are using the cyclic prefix (CP)-based orthogonal frequency division multiplexing (OFDM) modulation offering better robustness to multipath channel effects. However, the use of $\mathrm{CP}$ and the high side lobes of the rectangular pulse shape spectrum induce a loss of the spectral efficiency.

Filter bank-based multicarrier/offset quadrature amplitude modulation (FBMC/OQAM) modulations are potential promising candidates for next generation systems [1] as well as 5G systems [2]. Indeed, the good frequency localization of the prototype filters [3-5] used in FBMC/OQAM offers to this latter the robustness to several impairments such as the timing misalignment between users [6].

*Correspondence: bouhadda.hanene@supcom.rnu.tn

${ }_{1}^{1}$ CNAM - CEDRIC/LAETITIA, 292 rue Saint-Martin, Paris 75003, France

2 SUP'COM - INNOV'COM - Carthage University, Route de Raoued, Ariana 2083,

Tunisia

Full list of author information is available at the end of the article
It is well known that multicarrier signals are constructed by a sum of $N$ independent streams transmitted over $N$ orthogonal subcarriers. Considering high values of $N$ and according to the central limit theorem [7], the superposition of these independent streams leads to a complex Gaussian multicarrier signal. For this reason, OFDM and FBMC/OQAM exhibit large peak-to-average power ratios (PAPR) [8-10], i.e., large fluctuations in their signal envelope, making both modulations very sensitive to nonlinear distortion (NLD) caused by a high-power amplifier (HPA).

The main objective of this paper is to study the bit error rate (BER) performance in the presence of memoryless NL HPA for both OFDM and FBMC/OQAM systems under additive white Gaussian noise (AWGN) and Rayleigh fading channels. A theoretical characterization of NLD effects on OFDM systems has been proposed in [11], where the authors focused on the impact of the nonlinear amplitude distortions induced by three HPA models: the soft envelope limiter (SEL), the solid state power amplifier (SSPA), and the traveling wave tube amplifier ( TWTA). In

\section{望 Springer}

(c) 2014 Bouhadda et al: licensee Springer. This is an Open Access article distributed under the terms of the Creative Commons Attribution License (http://creativecommons.org/licenses/by/2.0), which permits unrestricted use, distribution, and reproduction in any medium, provided the original work is properly credited. 
this paper, the authors, proposed a theoretical characterization of the NLD parameters. We note that, except for the SEL HPA model, the investigation presented in [11] gives only semi-analytical results which could not be easily extended to measured HPA. Other contributions $[12,13]$ used the results presented in [11] to study the effect of HPA on multiple-input multiple-output (MIMO) transmit diversity systems. The impact of the HPA on out-of-band spectral regrowth was also studied for the OFDM system $[14]$ as well as the FBMC/OQAM case $[9,15]$.

Our aim is to evaluate the impact of the in-band distortions on OFDM and FBMC/OQAM modulations when a memoryless NL HPA is used. It is worth noting that, to the best of our knowledge, this is supposed to be the first paper that investigates the theoretical BER performance of the nonlinearly amplified FBMC/OQAM systems. In another way, this work extends the results presented in [11] to any modeled or measured HPA. The polynomial fitting of the amplitude-amplitude modulation (AM/AM) and amplitude-phase modulation (AM/PM) conversion characteristics allows complete theoretical characterization of the nonlinear distortion parameters for both multicarrier modulation schemes.

We will first demonstrate that FBMC/OQAM signals are more sensitive to phase rotation than OFDM ones. Indeed, if no phase correction is done, or in the presence of phase estimation errors, the intrinsic interference in FBMC/OQAM will increase the error probability compared to the OFDM case. In the case of a perfect phase correction at the receiver side, both techniques exhibit the same performance. Analytical closed-form expressions of the BER are established based on polynomial decomposition of the HPA NL characteristics. We notice that the proposed method can be applied for any memoryless HPA model and even for real measured ones. In order to validate the obtained BER expressions, various comparisons are made with respect to simulation results.

The rest of this paper is organized as follows: Section 2 describes the system model with brief introduction to OFDM and FBMC/OQAM modulations in addition to some elementary information on commonly used memoryless HPA models, exhibiting AM/AM and AM/PM distortions. In Sections 3 and 4, we present the theoretical model and the estimation of NLD parameters. Further, we develop a theoretical analysis of the BER for both OFDM and FBMC/OQAM systems in Section 5 . The obtained BER expressions are then evaluated through various simulation results, which are presented in Section 6. Finally, Section 7 gives the conclusion of this work.

\section{System model}

In this paper, we consider a FBMC/OQAM and OFDM transceiver with memoryless NL HPA as shown in Figure 1. We investigate the impact of NLD on BER performance in the case of AWGN and fading Rayleigh channels. We underline that all results can be generalized to frequency-selective channel. In the following, we will discuss the architecture of the OFDM and FBMC/OQAM systems. Then, we will introduce the commonly used HPA models in the literature.

\subsection{Introduction to OFDM and FBMC/OQAM 2.1.1 OFDM}

In OFDM system, bits are mapped to constellation symbols where the modulation and demodulation are, respectively, insured by the inverse fast Fourier transform (IFFT) and the fast Fourier transform (FFT). The time domain of an OFDM symbol calculated with $N$ IFFT point is given by

$$
i(t)=\sum_{n=-\infty}^{+\infty} \sum_{m=0}^{N-1} c_{m, n} f(t-n T) e^{j \frac{2 \pi}{T} m t}
$$

where

- $N$ is the number of subcarriers,

- $T$ is the OFDM symbol period,

- $c_{m, n}$ is a complex-valued symbol transmitted on the $m$ th subcarrier and at the instant $n T$, and

- $f(t)$ is a rectangular time window, defined by

$$
f(t)=\left\{\begin{array}{ll}
\frac{1}{\sqrt{T}} & t \in[0, T] \\
0 & \text { elsewhere }
\end{array} .\right.
$$

Considering high values of $N$ and according to the central limit theorem [7], the IFFT block transforms a set of independent complex random variables to a set of complex Gaussian random ones.

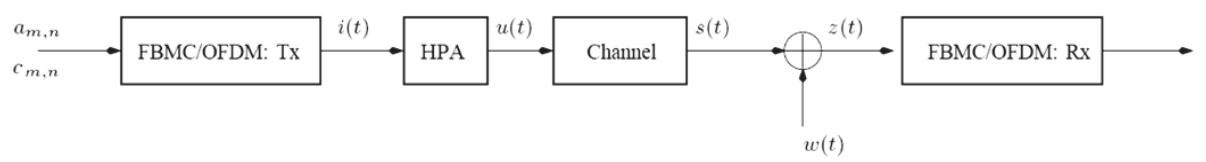

Figure 1 The transmission system model with FBMC/OQAM and OFDM modulations. 
In a distortion-free noiseless channel, the received symbol is given by the following equation

$$
\begin{aligned}
y_{m_{0}, n_{0}}= & \widehat{c}_{m_{0}, n_{0}}=\left\langle i(t), f\left(t-n_{0} T\right) e^{j \frac{2 \pi}{T} m_{0} t}\right\rangle \\
= & \int_{-\infty}^{+\infty} i(t) f\left(t-n_{0} T\right) e^{-j \frac{2 \pi}{T} m_{0} t} d t \\
= & \sum_{n=-\infty}^{+\infty} \sum_{m=0}^{N-1} \int_{-\infty}^{+\infty} c_{m, n} f(t-n T) f\left(t-n_{0} T\right) e^{j \frac{2 \pi}{T}\left(m-m_{0}\right) t} d t
\end{aligned}
$$

where

- $\widehat{c}_{m_{0}, n_{0}}$ is the received symbol, and

- $\langle.,$.$\rangle stands for the inner product.$

\subsubsection{FBMC/OQAM}

The considered FBMC/OQAM system is transmitting offset quadrature amplitude modulation (OQAM) symbols $[4,16]$, where the in-phase and the quadrature components are time staggered by half a symbol period, $T / 2$. Moreover, for two adjacent subcarriers, if we consider that the time delay $T / 2$ is introduced into the imaginary part of the QAM symbols on one of the subcarriers, then it is introduced into the real part of the symbols on the other one as shown on Figure $2 b$. Accordingly, the baseband continuous-time model of the FBMC/OQAM transmitted signal can be defined as follows [4]:

$$
i(t)=\sum_{n=-\infty}^{+\infty} \sum_{m=0}^{N-1} a_{m, n} h(t-n T / 2) e^{j \frac{2 \pi}{T} m t} e^{j \varphi_{m, n}}
$$

where

- $a_{m, n}$ is a real symbol transmitted on the $m$ th subcarrier and at the instant $n T$,

- $h(t)$ is the prototype filter impulse response, and

- $\varphi_{m, n}$ is the phase term which is given by

$$
\varphi_{m, n}=\frac{\pi}{2}(m+n)-\pi m n .
$$

Let $\gamma_{m, n}(t)$ be a time and frequency shifted version of $h(t)$

$$
\gamma_{m, n}(t)=h(t-n T / 2) e^{j \frac{2 \pi}{T} m t} e^{j \varphi_{m, n}} .
$$

Then, we can rewrite Equation 3 as follows:

$$
i(t)=\sum_{m=0}^{N-1} \sum_{n=-\infty}^{+\infty} a_{m, n} \gamma_{m, n}(t)
$$

In a distortion-free noiseless channel, the demodulated signal $y_{m_{0}, n_{0}}$ at time instant $n_{0}$ and subcarrier $m_{0}$ is given by

$$
\begin{aligned}
y_{m_{0}, n_{0}} & =\left\langle i(t), \gamma_{m_{0}, n_{0}}(t)\right\rangle=\int_{-\infty}^{+\infty} i(t) \gamma_{m_{0}, n_{0}}^{*}(t) d t \\
& =\sum_{n=-\infty}^{+\infty} \sum_{m=0}^{N-1} a_{m, n} \int_{-\infty}^{+\infty} \gamma_{m, n}(t) \gamma_{m_{0}, n_{0}}^{*}(t) d t \\
& =a_{m_{0}, n_{0}}+\sum_{n} \sum_{m} \int_{(m, n) \neq\left(m_{0}, n_{0}\right)}^{+\infty} \gamma_{m, n}(t) \gamma_{m_{0}, n_{0}}^{*}(t) d t
\end{aligned}
$$

where $\gamma_{m_{0}, n_{0}}^{*}(t)$ is the complex conjugate of $\gamma_{m_{0}, n_{0}}(t)$.

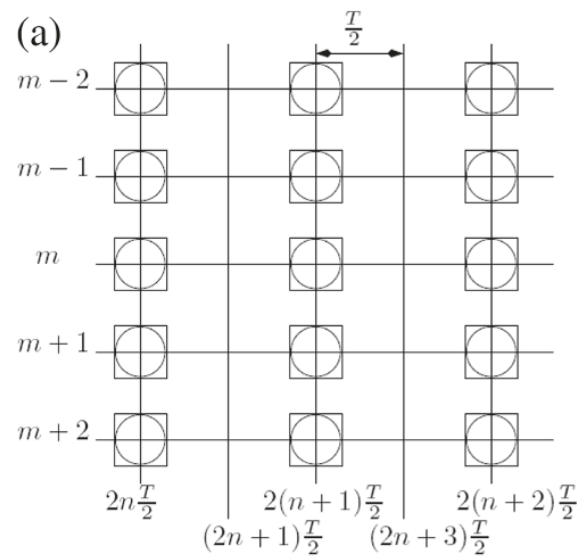

Real part of QAM symbol

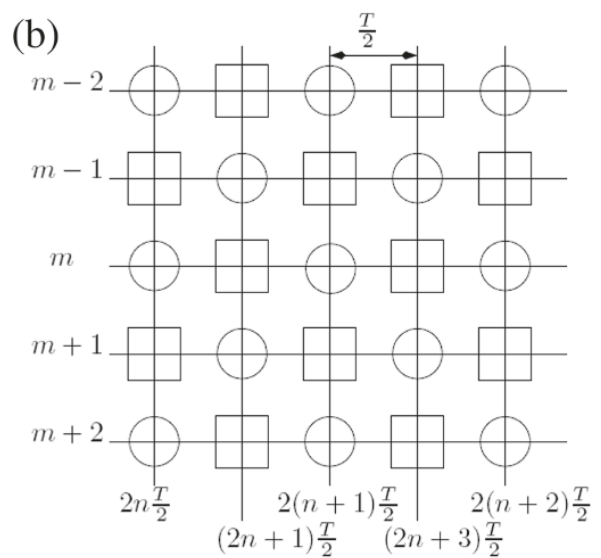

Imaginary part of QAM symbol

Figure 2 FBMC/OQAM and OFDM symbol mapping on subcarriers. (a) OFDM and (b) FBMC. 
According to [5], the prototype filter is designed such that the intrinsic interference term is orthogonal to the useful symbol, i.e., it is purely imaginary.

$$
j u_{m_{0}, n_{0}}=\sum_{n} \sum_{m} \underbrace{\int_{(m, n) \neq\left(m_{0}, n_{0}\right)}^{+\infty} \gamma_{m, n}(t) \gamma_{m_{0}, n_{0}}^{*}(t) d t}_{\Psi_{\Delta m, \Delta n}}
$$

Considering the PHYDYAS prototype filter proposed in [5], the coefficients $\Psi_{\Delta m, \Delta n}$ are given in Table 1. Consequently, a nearly perfect reconstruction of the transmitted real symbols $a_{m, n}$ is obtained by taking the real part (OQAM decision) of the demodulated signal $y_{m_{0}, n_{0}}$.

\subsection{HPA model}

A HPA model or a real measured one can be entirely described by its input/output or transfer function characteristics. The AM/AM and AM/PM characteristics indicate the relationship between, respectively, the modulus and the phase variation of the output signal as functions of the modulus of the input one.

The OFDM (Equation 1) or FBMC/OQAM (Equation 3) modulated symbol stream has a complex envelop $i(t)=$ $x(t)+j y(t)$ that can be written for both modulation schemes as

$$
i(t)=\rho(t) e^{j \varphi(t)}
$$

where

- $\rho(t)=\sqrt{|x(t)|^{2}+|y(t)|^{2}}$ is the signal input modulus, and

- $\varphi(t)=\arctan \left(\frac{y(t)}{x(t)}\right)$ is the signal input phase.

For simplicity sake, we have discarded the time variable $t$ from $\rho$ and $\varphi$. Then, the amplified signal $u(t)$ can be written as

$$
\begin{aligned}
u(t) & =F_{a}(\rho) \exp \left(j F_{p}(\rho)\right) \exp (j \varphi) \\
& =S(\rho) \exp (j \varphi)
\end{aligned}
$$

where

- $F_{a}(\rho)$ is the AM/AM characteristic of the HPA,

- $F_{p}(\rho)$ is the AM/PM characteristic of the HPA, and

- $S(\rho)=F_{a}(\rho) \exp \left(j F_{p}(\rho)\right)$ is the complex soft envelop of the amplified signal $u(t)$.

Table 1 Transmultiplexer impulse response

\begin{tabular}{lrcrcccc}
\hline & $n_{0}-\mathbf{3}$ & $n_{0}-2$ & $n_{0}-1$ & $n_{0}$ & $n_{0}+1$ & $n_{0}+2$ & $n_{0}+3$ \\
\hline$m_{0}-1$ & $0.043 j$ & $0.125 j$ & $0.206 j$ & $0.239 j$ & $0.206 j$ & $0.125 j$ & $0.043 j$ \\
$m_{0}$ & $-0.067 j$ & 0 & $-0.564 j$ & 1 & $0.564 j$ & 0 & $0.067 j$ \\
$m_{0}+1$ & $0.043 j$ & $-0.125 j$ & $0.206 j$ & $-0.239 j$ & $-0.206 j$ & $-0.125 j$ & $0.043 j$ \\
\hline
\end{tabular}

Then, the signal $z(t)$ at the input of the demodulator can be written as

$$
z(t)=h_{c}(t) \otimes u(t)+w(t)
$$

where

- $h_{c}(t)$ is the channel impulse response, and

- $\otimes$ stands for the convolution product.

In our analysis, we will consider some memoryless HPA models that are commonly used in the literature.

\subsubsection{Soft envelope limiter (SEL)}

It is used for modeling a HPA with a perfect predistortion system. The global transfer function of the predistortion followed by the HPA is thus a limiter which can be described by the following AM/AM and AM/PM functions [17]:

$$
\begin{aligned}
& F_{a}(\rho)= \begin{cases}\rho, & \rho \leq A_{\text {sat }} \\
A_{\text {sat }}, & \rho>A_{\text {sat }}\end{cases} \\
& F_{p}(\rho)=0
\end{aligned}
$$

where $A_{\text {sat }}$ is the HPA input saturation level.

\subsubsection{Solid state power amplifier (SSPA)}

This model, also known as the Rapp model, was presented in [18] and presents only AM/AM conversion. It can be expressed as

$$
\begin{aligned}
& F_{a}(\rho)=\frac{\rho}{\left(1+\left(\frac{\rho}{A_{\text {sat }}}\right)^{2 v}\right)^{\frac{1}{2 v}}} \\
& F_{p}(\rho)=0
\end{aligned}
$$

where $v$ is a smoothness factor that controls the transition from the linear region to the saturation region, $(v>0)$. This HPA model assumes a linear performance for low amplitudes of the input signal. Then, a transition towards a constant saturated output is observed. When $v \rightarrow \infty$, the Rapp model converges towards the SEL.

\subsubsection{Traveling wave tube amplifier (TWTA)}

Saleh's model is generally used for modeling TWT amplifiers [19]. This HPA model has been mainly used in several works dealing with the impact of nonlinearities in OFDM systems $[20,21]$. According to this model, the AM/AM and $\mathrm{AM} / \mathrm{PM}$ conversion characteristics can be expressed as follows:

$$
\begin{aligned}
& F_{a}(\rho)=A_{\text {sat }}^{2} \frac{\rho}{\rho^{2}+A_{\text {sat }}^{2}} \\
& F_{p}(\rho)=\varphi_{0} \frac{\rho^{2}}{\rho^{2}+A_{\text {sat }}^{2}}
\end{aligned}
$$


where $\varphi_{0}$ controls the maximum phase distortion introduced by this HPA model.

The AM/AM and AM/PM characteristics cause distortions on the constellation scheme and spectral regrowth, degrading then the system performance.

In practice, in order to avoid or at least to reduce the effects of nonlinearities, the HPA is operated at a given input back-off (IBO) from a given level. We underline that two definitions for the IBO can be found in the literature. In the first definition, the IBO is computed from the 1- $\mathrm{dB}$ compression point [22]. However, in the second one [23], the authors defined the IBO form the input saturation level. In this paper, we will use the second definition, in which the IBO is defined as follows:

$$
\mathrm{IBO}=10 \log _{10}\left(\frac{A_{\mathrm{sat}}^{2}}{\sigma^{2}}\right)
$$

where $\sigma^{2}$ is the variance of the input signal (mean input signal power).

In Figure 3, we plot the AM/AM and AM/PM characteristics of SEL, SSPA, and TWTA models, where $A_{\text {sat }}=1$, $\varphi_{0}=\pi / 3$, and $v=1$.

(a)

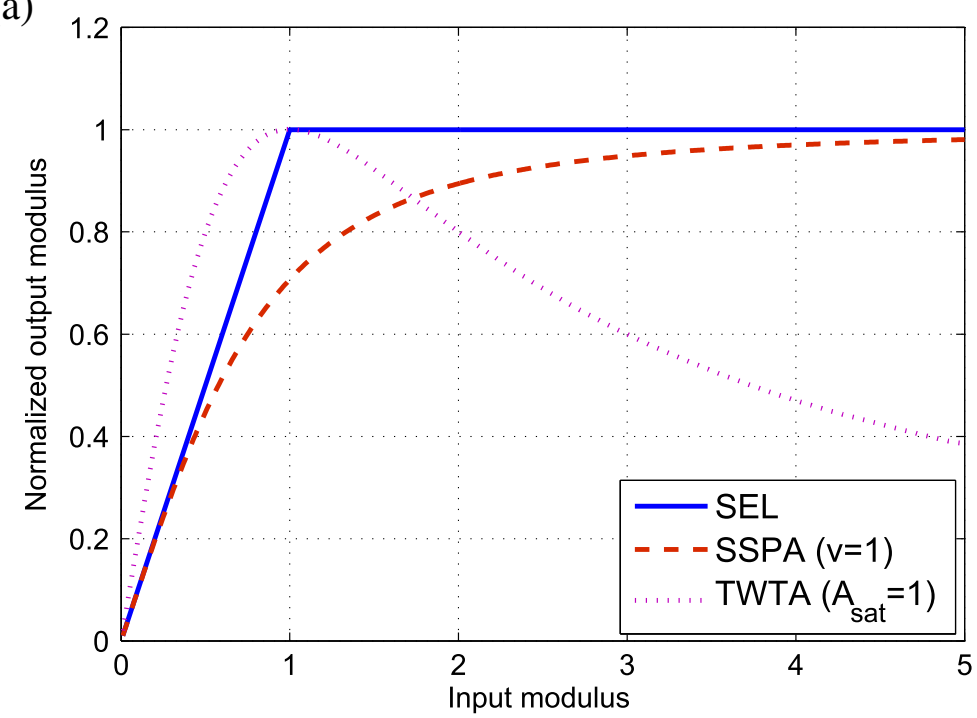

(b)

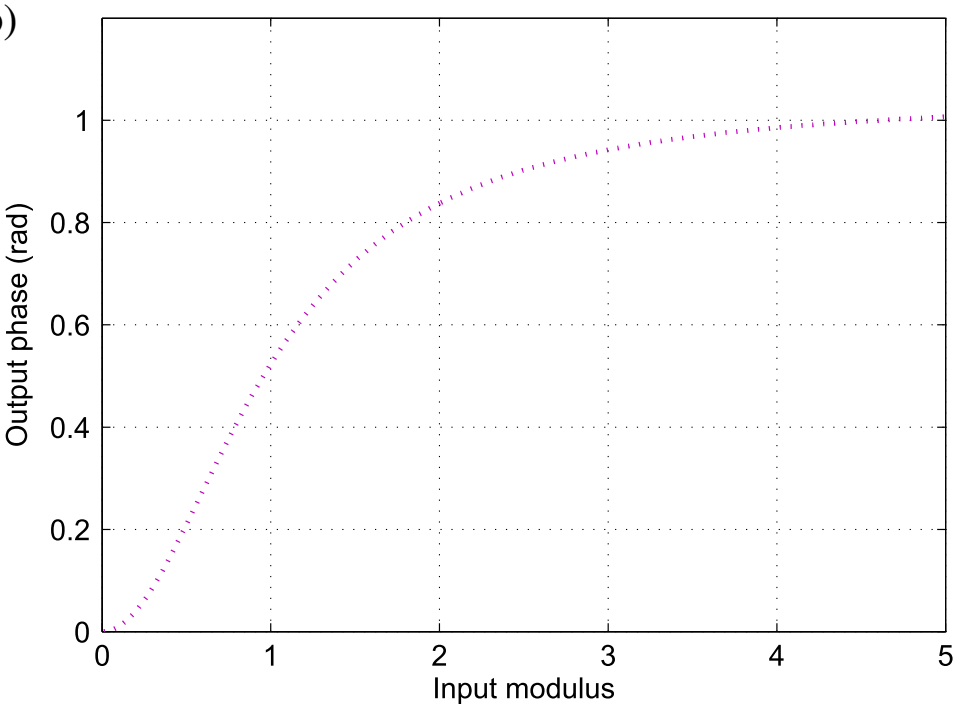

Figure 3 AM/AM and AM/PM characteristics of SEL, SSPA, and TWTA models. (a) AM/AM characteristics. (b) AM/PM characteristic of the TWTA $\left(A_{\text {sat }}=1, \varphi_{0}=\pi / 3\right)$. 


\section{Nonlinear distortion modeling}

When considering a large number of subcarriers $N$, the input signal $i(t)$ is assumed to be a zero mean, complex Gaussian random process. According to the Bussgang theorem $[17,24,25]$, the NL HPA output $u(t)$ is related to the input $i(t)$ by the following equation:

$$
u(t)=K(t) i(t)+d(t)
$$

where

- $d(t)$ is a zero mean noise, which is uncorrelated from $i(t)$.

- $K(t)$ is a complex gain with modulus $|K|$ and phase $\phi_{K}(t)$.

For a high number of subcarriers $N$, the mean of the complex Gaussian random process $i(t)$ tends to zero. In this case, $K(t)$ can be expanded as a Fourier series

$$
K(t)=\sum_{i=-\infty}^{+\infty} k_{i} e^{j 2 \pi i t / T}
$$

where $k_{i}$ is the Fourier coefficient given by

$$
k_{i}=\int_{-T / 2}^{T / 2} K(t) e^{-j 2 \pi i t / T} .
$$

The signal at the output of the HPA can be written as

$$
u(t)=\sum_{i=-\infty}^{+\infty} i(t) k_{i} e^{j 2 \pi i t / T}+d(t) .
$$

For both OFDM and FBMC/OQAM modulations, it is easy to verify that $k_{i}=0$ for $i>0$. We can write then the signal at the output of the HPA:

$$
u(t)=k_{0} i(t)+d(t)=K i(t)+d(t) .
$$

According to [11], $K$ can be computed analytically by

$$
K=\frac{1}{2} \mathbb{E}\left[\frac{\partial S(\rho)}{\partial \rho}+\frac{S(\rho)}{\rho}\right]
$$

where $\mathbb{E}$ is the expectation operator. We recall that $S(\rho)=$ $F_{a}(\rho) \exp \left(j F_{p}(\rho)\right)$ is the complex soft envelop of the amplified signal $u(t)$.

The variance $\sigma_{d}^{2}$ of the NL distortion $d(t)$ is given by the following equation:

$$
\sigma_{d}^{2}=\mathbb{E}\left(|d(t)|^{2}\right)=\mathbb{E}\left(|S(\rho)|^{2}\right)-|K|^{2} \mathbb{E}\left(\rho^{2}\right) .
$$

In Table 2, we compare the NLD parameter values given by Equations 20 and 21 for both OFDM and FBMC/OQAM by considering a TWT HPA model with $\varphi_{0}=\pi / 6$ and $A_{\text {sat }}=1$. The estimation of the parameters $K$ and $\sigma_{d}^{2}$ is made with $10^{6} 4$ QAM symbols using several values of subcarriers $N$. The comparison is made for two values of IBO of 4 and $8 \mathrm{~dB}$.

The results illustrated in Table 2 show that for a sufficiently high number of subcarriers $N$, the OFDM and FBMC/OQAM modulated signals, which are considered as Gaussian signals (according to the central limit theorem [7]), can be modeled by the same NLD parameters $K$ and $\sigma_{d}^{2}$ when they are passed through a given nonlinear HPA.

\section{Analytical computation of $K$ and $\sigma_{d}^{2}$}

The analytical computation of the NLD parameters $K$ and $\sigma_{d}^{2}$ depends on the complexity of the expression of $S(\rho)$. Indeed and based on Equations 20 and 21, the authors in [11] computed analytically $K$ and $\sigma_{d}^{2}$ in the case of the SEL HPA model. In this case, $K$ and $\sigma_{d}^{2}$ are expressed as

\begin{tabular}{|c|c|c|c|c|c|c|}
\hline \multirow[b]{2}{*}{$N$} & \multicolumn{2}{|c|}{ OFDM } & \multicolumn{2}{|c|}{ FBMC/OQAM } & \multicolumn{2}{|c|}{ Error } \\
\hline & $K$ & $\sigma_{d}^{2}$ & $K$ & $\sigma_{d}^{2}$ & $K$ & $\sigma_{d}^{2}$ \\
\hline \multicolumn{7}{|c|}{$\mathrm{IBO}=4 \mathrm{~dB}$} \\
\hline 4 & $0.5796+0.1137 j$ & $1.6392 \times 10^{-2}$ & $0.5792+0.1137 j$ & $1.6272 \times 10^{-2}$ & $1.6000 \times 10^{-7}$ & $1.4400 \times 10^{-7}$ \\
\hline 64 & $0.5912+0.1107 j$ & $1.1338 \times 10^{-2}$ & $0.5918+0.1107 j$ & $1.1339 \times 10^{-2}$ & $3.6000 \times 10^{-7}$ & $1.0000 \times 10^{-12}$ \\
\hline 1,024 & $0.5916+0.1106 j$ & $1.1339 \times 10^{-2}$ & $0.5923+0.1104 j$ & $1.1339 \times 10^{-2}$ & $5.3000 \times 10^{-7}$ & 0.0000 \\
\hline \multicolumn{7}{|c|}{$\mathrm{IBO}=8 \mathrm{~dB}$} \\
\hline 4 & $0.7662+0.0868 j$ & $7.0879 \times 10^{-3}$ & $0.7677+0.0858 j$ & $6.9633 \times 10^{-3}$ & $3.2500 \times 10^{-7}$ & $1.5525 \times 10^{-8}$ \\
\hline 64 & $0.7728+0.0830 j$ & $2.4249 \times 10^{-3}$ & $0.7728+0.0831 j$ & $2.4256 \times 10^{-3}$ & $1.0000 \times 10^{-8}$ & $4.9000 \times 10^{-13}$ \\
\hline 1,024 & $0.7727+0.0830 j$ & $2.4256 \times 10^{-3}$ & $0.7728+0.0830 j$ & $2.4258 \times 10^{-3}$ & $1.0000 \times 10^{-8}$ & $4.0000 \times 10^{-14}$ \\
\hline
\end{tabular}

$$
\begin{aligned}
& K=\left(1-e^{-\frac{A_{\text {sat }}^{2}}{\sigma^{2}}}\right)+\frac{1}{2} \sqrt{\pi \frac{A_{\text {sat }}^{2}}{\sigma^{2}}} \operatorname{erfc}\left(\sqrt{\frac{A_{\text {sat }}^{2}}{\sigma^{2}}}\right) \\
& \sigma_{d}^{2}=\sigma^{2}\left(1-e^{-\frac{A_{\text {sat }}^{2}}{\sigma^{2}}}-K^{2}\right) .
\end{aligned}
$$

Nevertheless, for more complicated expressions of $S(\rho)$, such as (12) and (13) (SSPA and TWTA models), the

Table 2 Comparison between estimated values of $K$ and $\sigma_{d}^{2}$ for both nonlinearly amplified OFDM and FBMC/OQAM signals 
derivation of analytical expressions for the parameters $K$ and $\sigma_{d}^{2}$ is intractable. In [11], no closed-form expression for $K$ and $\sigma_{d}^{2}$ is given for SSPA and TWTA HPA models. In order to simplify the computation and obtain analytical expressions for $K$ and $\sigma_{d}^{2}$ for any HPA model, we propose a polynomial approximation of $S(\rho)$. By doing this, we will be able to analytically compute the NLD parameters of Equations 20 and 21 for any HPA conversion characteristics after polynomial fitting.

\subsection{Proposed method}

Our approach aims to approximate the HPA conversion characteristics by a polynomial model [26], which can be written as

$$
u(t)=\sum_{n=1}^{L} a_{n} i(t)|i(t)|^{n-1}
$$

where

- $L$ is the polynomial order, and

- $a_{n}$ are the complex coefficients of the polynomial approximation.

In classical polynomial models existing in literature, only odd coefficients are generally used for fitting the AM/AM curve [14]. In order to better fit the complex soft envelope $S(\rho)$ of the amplified signal, we have chosen to use a full rank order polynomial with odd and even coefficients. By using the polynomial approximation of Equation 24, the new HPA output is given by

$$
\begin{aligned}
u(t) & =e^{j \varphi} \sum_{n=1}^{L} a_{n} \rho^{n} \\
& =e^{j \varphi} S(\rho)
\end{aligned}
$$

where the new expression of $S(\rho)$ is equal to

$$
S(\rho)=\sum_{n=1}^{L} a_{n} \rho^{n}
$$

The complex valued polynomial coefficients $a_{n}, n=1 . . L$ can be easily obtained by using a classical least square (LS) method [27].

\subsection{Analytical computation of $K$ and $\sigma_{d}^{2}$ using polynomial approximation}

Using the polynomial approximation of $S(\rho)$, we simplify the computation of $K$ and $\sigma_{d}^{2}$ given by Equations 20 and 21 . This will be achieved by the computation of the expectation of a power of Rayleigh random variable, $\mathbb{E}\left[\rho^{n}\right]$, where, $n=1 \ldots \ldots 2 L$. Equation 20 can be rewritten as

$$
K=\frac{1}{2} \sum_{n=1}^{L}(n+1) a_{n} \mathbb{E}\left[\rho^{n-1}\right] .
$$

The variance $\sigma_{d}^{2}$ of the NLD $d(t)$ given in (21) becomes

$$
\sigma_{d}^{2}=\sum_{n=1}^{L}\left|a_{n}\right|^{2} \mathbb{E}\left[\rho^{2 n}\right]+2 \sum_{n, l=1, n \neq l}^{L} \Re\left[a_{n} a_{l}\right] \mathbb{E}\left[\rho^{n+l}\right]-|K|^{2} \mathbb{E}\left[\rho^{2}\right]
$$

where $\mathfrak{R}[$.$] stands for the real part.$

The above theoretical expressions of $K$ and $\sigma_{d}^{2}$ involve the computation of the expectation of $\rho^{n}$ ( $n$ is a positive integer). This expectation is equivalent to calculate the $n$th derivation of the moment-generating function (MGF).

We can write $\mathbb{E}\left[\rho^{n}\right]$ as

$$
\mathbb{E}\left[\rho^{n}\right]=\left.\frac{\partial^{n} M(t)}{\partial t^{n}}\right|_{t=0}
$$

where

- $\mathrm{n}$ is a positive integer, and

- $M(t)$ is the MGF given by

$$
M(t)=e^{\rho t}
$$

A generic expression for the computation of $\mathbb{E}\left[\rho^{n}\right]$ is given in [27]. It is expressed as follows:

- For odd values of $n$, we have

$$
\begin{aligned}
\mathbb{E}\left[\rho^{n}\right] & =\left.\frac{\partial^{n} M(t)}{\partial t^{n}}\right|_{t=0} \\
& =\sqrt{\frac{\pi}{2}} \sigma^{n} \prod_{i=0}^{\frac{n-1}{2}}(2 i+1) .
\end{aligned}
$$

- For even values of $n$, we have

$$
\begin{aligned}
\mathbb{E}\left[\rho^{n}\right] & =\left.\frac{\partial^{n} M(t)}{\partial t^{n}}\right|_{t=0} \\
& =(\sqrt{2} \sigma)^{n}\left(\frac{n}{2}\right) !
\end{aligned}
$$

where ! stands for the factorial operator.

Using Equations 27, 31, and 32, we have finally for the NL parameter $K$

$$
\begin{aligned}
K= & \sqrt{\frac{\pi}{8}} \sum_{n=1, n \text { odd }}^{L}(n+1) a_{n} \sigma^{n} \prod_{i=0}^{\frac{n-1}{2}}(2 i+1) \\
& +\frac{1}{2} \sum_{n=2, n \text { even }}^{L}(n+1) a_{n}(\sqrt{2} \sigma)^{n}\left(\frac{n}{2}\right) !
\end{aligned}
$$


For the variance $\sigma_{d}^{2}$ of the NL noise $d(t)$, we obtain the following expression:

$$
\begin{aligned}
\sigma_{d}^{2}= & \sum_{n=1}^{L}\left|a_{n}\right|^{2} 2^{n} \sigma^{2 n} n !-2|K|^{2} \sigma^{2} \\
& +\sqrt{\frac{4 \pi}{2}} \sum_{n, l=1, n \neq l,(n+l) \text { odd }}^{L} \Re\left[a_{n} a_{l}\right] \sigma^{n+l} \prod_{i=0}^{\frac{n+l-1}{2}}(2 i+1) \\
& +2 \sum_{n, l=1, n \neq l,(n+1) \text { even }}^{L} \Re\left[a_{n} a_{l}\right](\sqrt{2} \sigma)^{n+l}\left(\frac{n+l}{2}\right) !
\end{aligned}
$$

We should note that both $K$ and $\sigma_{d}^{2}$ are function of the signal IBO. Indeed, the variance $\sigma$ of the signal at the input of the HPA can be written as

$$
\sigma=\frac{A_{\mathrm{sat}}}{\sqrt{10^{I B O / 10}}} .
$$

\subsubsection{Validation of the analytical expressions of $K$ and $\sigma_{d}^{2}$}

In order to validate our analytical analysis of $K$ and $\sigma_{d}^{2}$ (Equations 33 and 34), at first, we will compute by simulation the values of $K$ and $\sigma_{d}^{2}$ obtained, respectively, by Equations 20 and 21 for a given HPA model (SSPA or TWTA). After approximation of each HPA model with the polynomial model of order $L=10$, the parameters $K$ and $\sigma_{d}^{2}$ can be theoretically computed based on Equations 33 and 34 . Table 3 compares the simulated and theoretically computed values for different HPA models and different scenarios. The results are given for $10^{6}$ 4QAM symbols modulated over 64 subcarriers. For the TWTA model, we have $A_{\text {sat }}=1$, and for the SSPA one, $v$ is fixed to 1 .

According to Table 3, we observe, with a polynomial approximation of order $L=10$, a very good agreement between simulation and theoretical results for both $K$ and $\sigma_{d}^{2}$. As confirmed by the error criterion, there is a good accuracy of the analytical expressions proposed for the computation of $K$ and $\sigma_{d}^{2}$ with respect to the computation based on Equations 20 and 21.

\subsubsection{Influence of polynomial approximation order}

In Table 4, we compare the NLD parameter values given by Equations 33 and 34 with an FBMC/OQAM modulated signal and for different values of the polynomial approximation order $L$ (Equation 24). In this table, we consider a TWTA model with $\varphi_{0}=\pi / 6, A_{\text {sat }}=1$, and IBO $=8 \mathrm{~dB}$.

Results illustrated in Table 4 show that for a sufficiently high order of the polynomial approximation $L$, the theoretically estimated values of $K$ and $\sigma_{d}^{2}$ based on Equations 33 and 34 are stable and correspond to the numerically simulated ones (Equations 20 and 21). It must also be noted that the polynomial order $L$ can be chosen very high without additional complexity. Indeed, as soon as coefficients $a_{n}$ are computed for a given HPA (using a classical least squares method), the computation of $K$ and $\sigma_{d}^{2}$ is straightforward using Equations 33 and 34 .

\section{Theoretical performance analysis}

The received signal after HPA and channel filtering can be expressed, after substituting $u(t)$ in Equation 10 by the expression of Equation 15, as

$$
z(t)=i(t) \otimes\left[K h_{c}(t)\right]+d(t) \otimes h_{c}(t)+w(t) .
$$

Looking at Equation 36, it is clear that the effect of the NL factor $K$ will be taken into account during frequency equalization at the receiver side $\left(h_{c}(t)\right.$ and $K$ will be estimated jointly). Nevertheless, in order to stress the impact of the phase rotation on both OFDM and FBMC/OQAM

\begin{tabular}{|c|c|c|c|c|c|c|c|}
\hline \multirow[b]{2}{*}{ IBO (dB) } & \multirow[b]{2}{*}{$\varphi_{0}$} & \multicolumn{2}{|c|}{ Simulation } & \multicolumn{2}{|c|}{ Theoretical } & \multicolumn{2}{|c|}{ Error } \\
\hline & & $K$ (Equation 20) & $\sigma_{d}^{2}$ (Equation 21) & $K$ (Equation 33) & $\sigma_{d}^{2}$ (Equation 34) & $K$ & $\sigma_{d}^{2}$ \\
\hline \multicolumn{8}{|l|}{ SSPA } \\
\hline 4 & - & 0.7699 & $4.4759 \times 10^{-3}$ & 0.7690 & $4.4431 \times 10^{-3}$ & $6.7240 \times 10^{-7}$ & $9.5481 \times 10^{-10}$ \\
\hline 6 & - & 0.8307 & $1.8950 \times 10^{-3}$ & 0.8297 & $1.8722 \times 10^{-3}$ & $1.1025 \times 10^{-6}$ & $7.0560 \times 10^{-11}$ \\
\hline 8 & - & 0.8798 & $7.0742 \times 10^{-4}$ & 0.8785 & $7.0583 \times 10^{-4}$ & $1.6129 \times 10^{-6}$ & $1.6384 \times 10^{-12}$ \\
\hline \multicolumn{8}{|l|}{ TWTA } \\
\hline 4 & 0 & 0.6042 & $1.0330 \times 10^{-2}$ & 0.6036 & $1.0317 \times 10^{-2}$ & $1.9360 \times 10^{-7}$ & $1.0609 \times 10^{-8}$ \\
\hline 6 & 0 & 0.6976 & $4.9589 \times 10^{-3}$ & 0.6969 & $4.9654 \times 10^{-3}$ & $4.9000 \times 10^{-7}$ & $1.2532 \times 10^{-9}$ \\
\hline 8 & 0 & 0.7784 & $2.1228 \times 10^{-3}$ & 0.7775 & $2.1030 \times 10^{-3}$ & $7.9210 \times 10^{-7}$ & $1.9600 \times 10^{-12}$ \\
\hline 4 & $\pi / 6$ & $0.5917+0.1106 j$ & $1.1339 \times 10^{-2}$ & $0.5904+0.1068 j$ & $1.1271 \times 10^{-2}$ & $1.6590 \times 10^{-5}$ & $5.3290 \times 10^{-9}$ \\
\hline 6 & $\pi / 6$ & $0.6887+0.0995 j$ & $5.5901 \times 10^{-3}$ & $0.6870+0.0948 j$ & $5.5812 \times 10^{-3}$ & $2.4324 \times 10^{-5}$ & $2.3104 \times 10^{-10}$ \\
\hline 8 & $\pi / 6$ & $0.7727+0.0830 j$ & $2.4194 \times 10^{-3}$ & $0.7727+0.0830 j$ & $2.4345 \times 10^{-3}$ & $3.5527 \times 10^{-5}$ & $2.2801 \times 10^{-10}$ \\
\hline
\end{tabular}
performances, we will present in Section 5.2 an analysis of the BER when the channel is an AWGN one and when no correction is made for the NL factor $K$. Within the same

Table 3 Comparison between numerical and theoretical values of $K$ and $\sigma_{d}^{2}$ 
Table 4 Impact of the polynomial order approximation on the estimated values of $K$ and $\sigma_{d}^{2}$

\begin{tabular}{|c|c|c|c|c|}
\hline \multirow[b]{2}{*}{$L$} & \multicolumn{2}{|c|}{ FBMC/OQAM } & \multicolumn{2}{|c|}{ Error } \\
\hline & $K$ & $\sigma_{d}^{2}$ & $K$ & $\sigma_{d}^{2}$ \\
\hline 5 & $0.7976+0.1570 j$ & $3.1941 \times 10^{-3}$ & $6.0985 \times 10^{-3}$ & $5.9259 \times 10^{-7}$ \\
\hline 7 & $0.8248+0.0713 j$ & $3.8676 \times 10^{-3}$ & $2.8651 \times 10^{-3}$ & $2.0831 \times 10^{-6}$ \\
\hline 10 & $0.7728+0.0830 j$ & $2.4345 \times 10^{-3}$ & $3.5527 \times 10^{-5}$ & $2.2801 \times 10^{-10}$ \\
\hline 15 & $0.7728+0.0830 j$ & $2.4345 \times 10^{-3}$ & $3.5527 \times 10^{-5}$ & $2.2801 \times 10^{-10}$ \\
\hline 20 & $0.7728+0.0830 j$ & $2.4345 \times 10^{-3}$ & $3.5527 \times 10^{-5}$ & $2.2801 \times 10^{-10}$ \\
\hline 30 & $0.7728+0.0830 j$ & $2.4345 \times 10^{-3}$ & $3.5527 \times 10^{-5}$ & $2.2801 \times 10^{-10}$ \\
\hline 100 & $0.7728+0.0830 j$ & $2.4345 \times 10^{-3}$ & $3.5527 \times 10^{-5}$ & $2.2801 \times 10^{-10}$ \\
\hline TWTA model & $0.7728+0.0830 j$ & $2.4345 \times 10^{-3}$ & & \\
\hline
\end{tabular}

section, we will evaluate theoretical BER in an AWGN channel with correction of the phase rotation related to the factor $K$. Section 5.3 is dedicated to the BER analysis of OFDM and FBMC/OQAM modulations in the case of the Rayleigh channel.

\subsection{Sensitivity of OFDM and FBMC/OQAM to phase error}

For simplicity reasons, we will conduct our analysis for 4QAM modulated symbols. The extension of this analysis to $\mathrm{M}-\mathrm{AM}(M>4)$ is straightforward.

\subsubsection{OFDM case}

The output constellation after HPA and OFDM demodulation is presented in Figure 4.

The distance $d$ (referred to as $d_{\text {OFDM }}$ in this case) is a function of the NLD induced by the HPA. It represents the distance projected on the real axis of the received signal

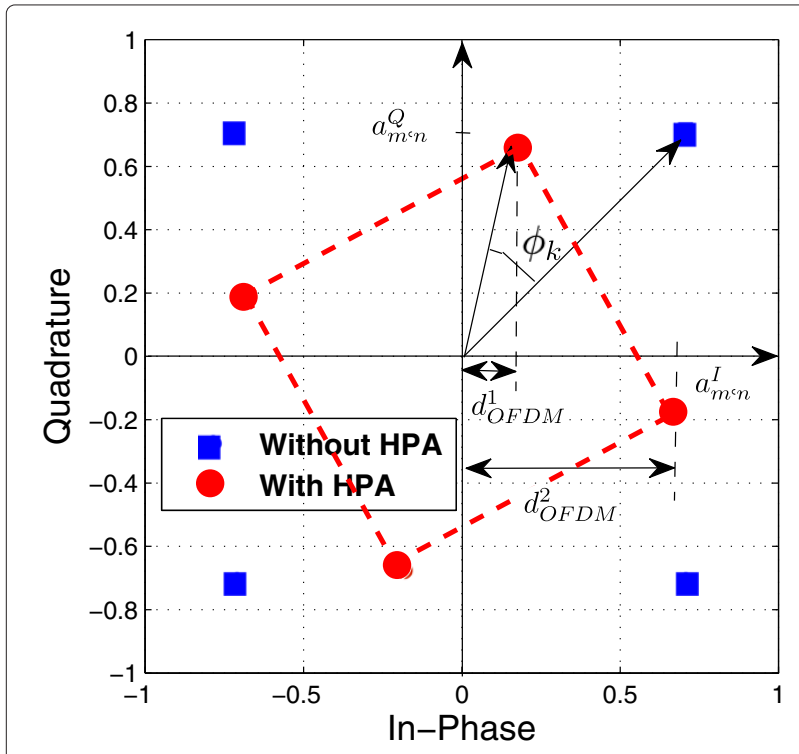

Figure 4 Impact of a phase rotation $\phi_{k}$ on OFDM constellation. points affected by the phase rotation. The distance $d$ is equal to

$$
d_{\mathrm{OFDM}}=\operatorname{Re}\left(\left(a_{m, n}^{I}+j a_{m, n}^{Q}\right)|K| \exp \left(j \phi_{k}\right)\right)
$$

where $a_{m, n}^{I}$ and $a_{m, n}^{Q}$ denote, respectively, the in-phase and the quadrature components of the transmitted complex symbol.

\subsubsection{FBMC/OQAM case}

With this modulation technique, the transmission of a real symbol on subcarrier $m_{0}$ generates a pure imaginary intrinsic interference $u_{m_{0}, n_{0}}$.

However, in the presence of a phase offset $\left(\phi_{k}\right)$, this interference is no longer imaginary. Consequently, by taking the real part of the received signal, we obtain a part of the useful signal distorted by the interference signal $\left(u_{m_{0}, n_{0}}\right)$.

Figure 5a presents the possible received constellation when the real part of the transmitted symbol $a_{m_{0}, n_{0}}$ is equal to 1 and when there is no phase rotation related to the channel or the HPA. The different points of the vertical line correspond to a real part $a_{m_{0}, n_{0}}=1$ and an imaginary part $u_{m_{0}, n_{0}}$ related to all possible values of adjacent symbols in frequency and time (see Figure $2 b$ ). We recall that the expression of $u_{m_{0}, n_{0}}$ is given by Equation 7 .

When there is no phase rotation related to the channel or the HPA, taking the real part of $y_{m_{0}, n_{0}}$ gives the corresponding real symbol $a_{m_{0}, n_{0}}$ free of interference. In the case of a phase rotation $\phi_{k}$ induced by the channel or the HPA, the received constellation of Figure 5a converts to that of Figure $5 \mathrm{~b}$. When taking the real part of $y_{m_{0}, n_{0}}$, it is obvious that the interference term $u_{m_{0}, n_{0}}$ will impact the decision process.

Figure 6 shows the 4QAM constellation affected by the HPA. It has to be noted that this constellation is considered before the OQAM demodulation. In this case, the $\mathrm{FBMC} / \mathrm{OQAM}$ distance $d_{\mathrm{FBMC}}$ is given by 


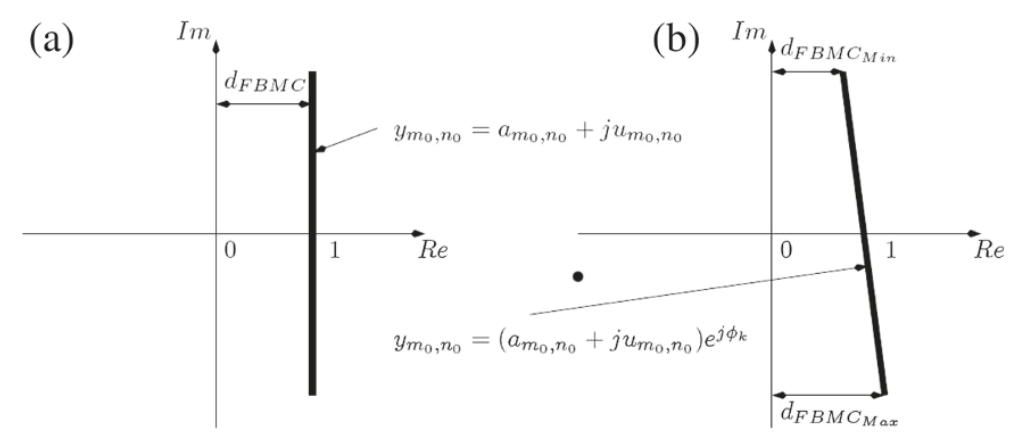

Figure $5 \mathrm{FBMC/OQAM}$ constellations with $a_{m_{0}, n_{0}}=1$. (a) $\phi_{k}=0$ and (b) $\phi_{k} \neq 0$.

$$
d_{\mathrm{FBMC}}=|K|\left(a_{m, n} \cos \left(\phi_{k}\right)-u_{m, n} \sin \left(\phi_{k}\right)\right) .
$$

$u_{m, n}$ is given by Equation 7, and it corresponds to all possible combinations of adjacent symbols.

\subsection{Performance analysis in the case of an AWGN channel} If no correction is made at the receiver side for the multiplicative constant $K$, the 4QAM BER in an AWGN channel is defined as follows [28]:

$\mathrm{BER}_{4 \mathrm{QAM}}^{\mathrm{AWGN}, \mathrm{K} \text { not corrected for }}=\frac{1}{2} \int_{0}^{+\infty} \operatorname{erfc}\left(\sqrt{\left(\frac{u^{2}}{4 N_{0}}\right)}\right) \operatorname{pdf}(u) d u$ where

- $u=\frac{d_{\min }}{2}$ is the decision distance $\left(d_{\min }\right.$, being the minimum distance),

- $\operatorname{pdf}(u)$ is the probability density function of $u$, and

- $N_{0} / 2$ is the power spectral density of the additive noise.

The power spectral density $N_{0}$ is given by

$$
N_{0}=\left(\sigma_{w}^{2}+\sigma_{d}^{2}\right) 2 T
$$

where

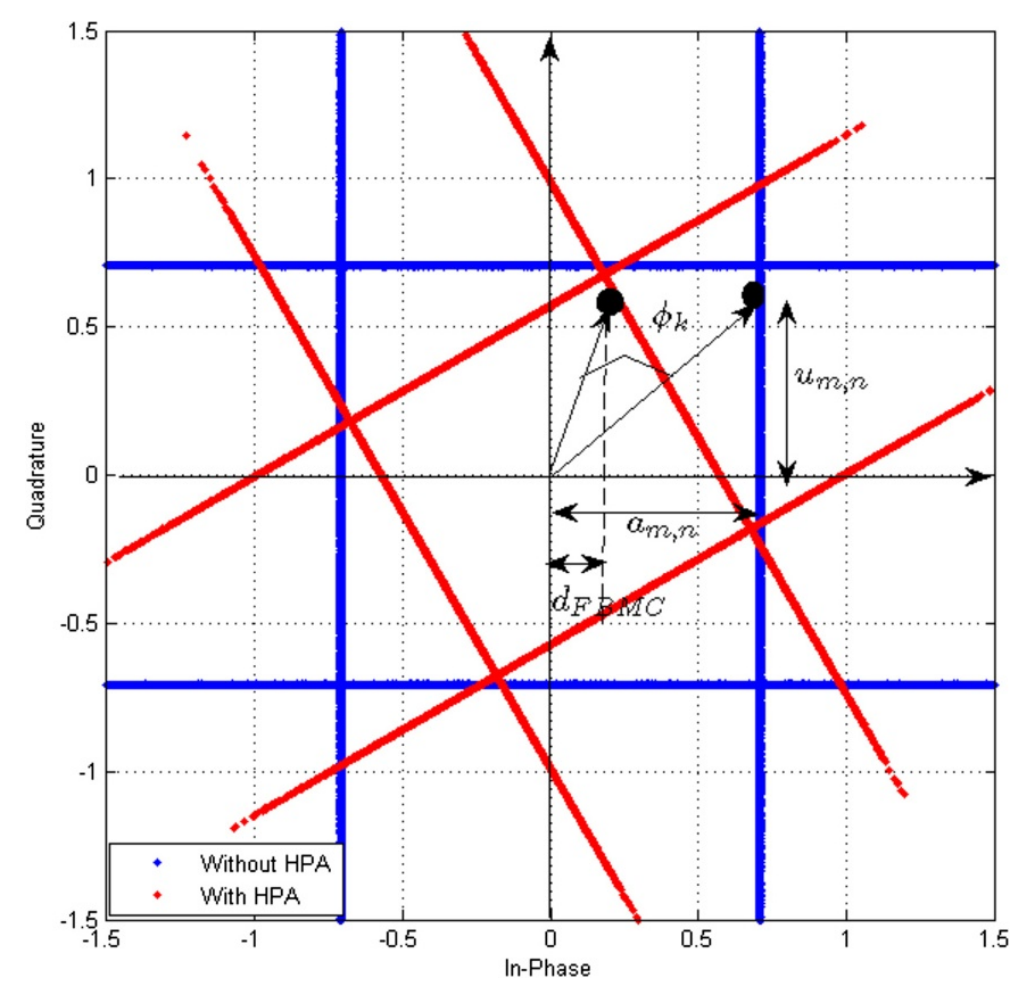

Figure 6 Impact of a phase rotation $\phi_{k}$ on FBMC/OQAM constellation. 
- $\sigma_{d}^{2}$ is the variance of the NL distortion $d(t)$, and

- $T$ is the symbol duration.

Equation 41 becomes so

$\mathrm{BER}_{4 \mathrm{QAM}}^{\mathrm{AWGN}, \mathrm{K} \text { not corrected for }}$

$$
=\frac{1}{2} \int_{0}^{+\infty} \operatorname{erfc}\left(\sqrt{\left(\frac{u^{2}}{2 T\left(\sigma_{w}^{2}+\sigma_{d}^{2}\right)}\right)}\right) \operatorname{pdf}(u) d u .
$$

In the case where we assume a perfect estimation of the NLD parameter $K$, after compensation for this factor, at the receiver side, we can rewrite the signal at the input of OFDM and FBMC/OQAM Rx as

$$
z(t)=i(t)+(d(t)+w(t)) \frac{1}{K} .
$$

For an $M$-ary quadrature amplitude modulation (MQAM), the BER after compensation for the factor $K$ can be written as follows [28]:

$\mathrm{BER}_{\mathrm{MQAN}, \mathrm{K} \text { corrected for }}^{\mathrm{AW}}$

$$
=\frac{2(\sqrt{M}-1)}{\sqrt{M} \log _{2}(M)} \operatorname{erfc}\left(\sqrt{\left(\frac{3 \log _{2}(M) E_{b a v g}}{2(M-1) N_{0}}\right)}\right)
$$

where

- $M$ is the alphabet of the modulation,

- $E_{\text {bavg }}$ is the average energy per bit normalized to 1 , and

- $N_{0} / 2$ is the power spectral density of the additive noise.

From Equation 42, it can be clearly seen that the power spectral density $N_{0}$, after correction of the NL factor $K$, is given by

$$
N_{0}=\frac{\left(\sigma_{w}^{2}+\sigma_{d}^{2}\right)}{|K|^{2}} 2 T
$$

We can finally rewrite Equation 43 as follows:

$$
\begin{aligned}
& \text { BER AWGN, K corrected for } \\
& \quad=\frac{2(\sqrt{M}-1)}{\sqrt{M} \log _{2}(M)} \operatorname{erfc}\left(\sqrt{\left(\frac{3 \log _{2}(M)|K|^{2}}{4(M-1) T\left(\sigma_{w}^{2}+\sigma_{d}^{2}\right)}\right)}\right) .
\end{aligned}
$$

\subsection{BER analysis with phase rotation correction in the case of a Rayleigh channel}

We assume a slowly varying flat fading Rayleigh channel with a coherence time larger than the symbol duration.
The Rayleigh fading amplitude $\alpha$ follows the probability density function (pdf)

$$
p_{\alpha}(\alpha)=\frac{\alpha}{\Omega} e^{\left(-\alpha^{2} / \Omega\right)}
$$

where $\Omega=\mathbb{E}\left[\alpha^{2}\right]$ is the average fading power.

Also, its power $\alpha^{2}$ is an exponentially distributed random variable with a pdf expressed as

$$
p_{\alpha^{2}}(\alpha)=\frac{1}{\Omega} e^{(-\alpha / \Omega)}
$$

The nonlinearly amplified signal in a Rayleigh fading channel, with coherent detection, is given by Equation 36 . It is expressed as

$$
\frac{h_{c}^{*}}{\left|h_{c}\right|^{2} K} z(t)=i(t)+\frac{d(t)}{K}+\frac{h_{c}^{*}}{\left|h_{c}\right|^{2} K} w(t)
$$

Then, the instantaneous signal-to-noise ratio (SNR) at the receiver can be expressed as

$$
\gamma=\gamma_{c} \frac{\alpha^{2}}{\alpha^{2} \sigma_{d}^{2}+\sigma_{w}^{2}}
$$

where $\gamma_{c}=|K|^{2} E_{b}$ and $E_{b}$ is the energy per bit.

Derivation of the pdf of $\gamma$

Lemma 1. Let $X=\alpha^{2}$ be an exponentially distributed random variable, then the SNR can be described as

$$
\gamma=\gamma_{c} \frac{X}{X \sigma_{d}^{2}+\sigma_{w}^{2}}=h(X)
$$

$h(X)$ is strictly increasing continuously differentiable function with inverse $X=g(\gamma)$. Then, $\gamma=h(X)$ is continuous with probability density function $p_{\gamma}(\gamma)$ given by [29]

$$
p_{\gamma}(\gamma)=p_{X}(g(\gamma)) g^{\prime}(\gamma)
$$

where $g(\gamma)=\sigma_{w}{ }^{2} \gamma /\left(\gamma_{c}-\sigma_{d}^{2} \gamma\right)$.

Then,

$p_{\gamma}(\gamma)= \begin{cases}\frac{\sigma_{w}^{2} \gamma_{c}}{\Omega\left(\gamma_{c}-\sigma_{d}^{2} \gamma\right)^{2}} e^{-\frac{\sigma_{w}^{2} \gamma}{\Omega\left(\gamma_{c}-\sigma_{d}^{2} \gamma\right)},}, \text { if } 0 \leq \gamma<\frac{\gamma_{c}}{\sigma_{d}^{2}} \\ 0 & \text { otherwise }\end{cases}$

The average BER can be calculated by averaging BER for instantaneous SNR over the distribution given in Equation 52. For such MQAM modulation, the BER can be found using

$$
\operatorname{BER}=\int_{0}^{\frac{\gamma c}{\sigma_{d}^{2}}} \operatorname{BER}(\gamma) p_{\gamma}(\gamma) d \gamma
$$


Substituting the $p d f p_{\gamma}(\gamma)$ of Equation 52 in the integral of Equation 53 gives

$$
\begin{aligned}
\operatorname{BER}_{\text {MQAM }}^{\text {Rayleigh }}= & \int_{0}^{\frac{\gamma_{c}}{\sigma d^{2}}} \frac{2(\sqrt{M}-1)}{\sqrt{M} \log _{2}(M)} \\
& \times \operatorname{erfc}\left(\sqrt{\left(\frac{3 \log _{2}(M) \gamma}{(M-1)}\right)}\right) \\
& \times \frac{\sigma_{w}^{2} \gamma_{c}}{\Omega\left(\gamma_{c}-\sigma_{d^{2}} \gamma\right)^{2}} e^{-\frac{\sigma_{w}^{2} \gamma}{\Omega\left(\gamma_{c}-\sigma_{d}^{2} \gamma\right)}} d \gamma .
\end{aligned}
$$

\section{Simulation results}

In this section, we present numerical results illustrating the impact of memoryless HPA nonlinearity on the performance of FBMC/OQAM and OFDM systems under AWGN and Rayleigh fading channels. In this work, we have considered FBMC/OQAM and OFDM systems with $N=64$ subcarriers transmitting M-QAM modulated symbols.

The BER is computed by averaging on $5 \times 10^{7}$ randomly generated FBMC/OQAM and OFDM symbols. We will investigate the cases where transmission is achieved through AWGN and Rayleigh fading channels. For both OFDM and FBMC/OQAM systems, we have considered three scenarios. In the first one, a SEL HPA model is used. In the second scenario, a TWT HPA model with only AM/AM distortion is considered. In the last scenario, a TWT HPA model is used, exhibiting both AM/AM and $\mathrm{AM} / \mathrm{PM}$ distortions. In all the simulations, the curve referred by 'linear' in the legend corresponds to the case when the power amplifier is perfectly linear.

\subsection{Impact of phase error on OFDM and FBMC/OQAM}

In Figure 7, the impact of the phase distortion on the BER of the considered OFDM and FBMC/OQAM systems is shown. This comparison was made for 4QAM modulated symbols at an $\mathrm{IBO}=8 \mathrm{~dB}$.

According to Figure 7, we observe that in the case where $\varphi_{0}=0$, the BER of OFDM and FBMC/OQAM systems are the same. This case corresponds to no phase rotation in the received constellation of Figures 4 and 6. However, by introducing a phase distortion with $\varphi_{0}=\pi / 3$, a significant gap appears between the BER of OFDM and FBMC/OQAM systems. In this case, the BER is computed using Equation 41. The pdf of the decision distance has been estimated by simulations and is given by Figure 8 . This pdf corresponds to the transmission of 4QAM symbols on FBMC/OQAM modulator using the PHYDYAS prototype filter [5].

The gap between the BER of the two modulation techniques can be explained by the intrinsic interference term introduced by the FBMC/OQAM. As an example, for $\mathrm{IBO}=4 \mathrm{~dB}, \varphi_{0}=\pi / 3$, and a BER $=5 \times 10^{-3}$, FBMC/OQAM modulation shows a loss of $\approx 7 \mathrm{~dB}$ in SNR compared to the OFDM-based one. Then, when considering a selective frequency channel, an error in estimating the phase rotation of the channel will have a bigger impact in FBMC/OQAM than in OFDM due its higher sensitivity.

\subsection{BER analysis in the case of an AWGN channel}

Using Equation 45, we first compare theoretical and simulation results for both OFDM and FBMC/OQAM in the case of 16QAM modulated symbols and transmitted over an AWGN channel. For the results, we assume a perfect

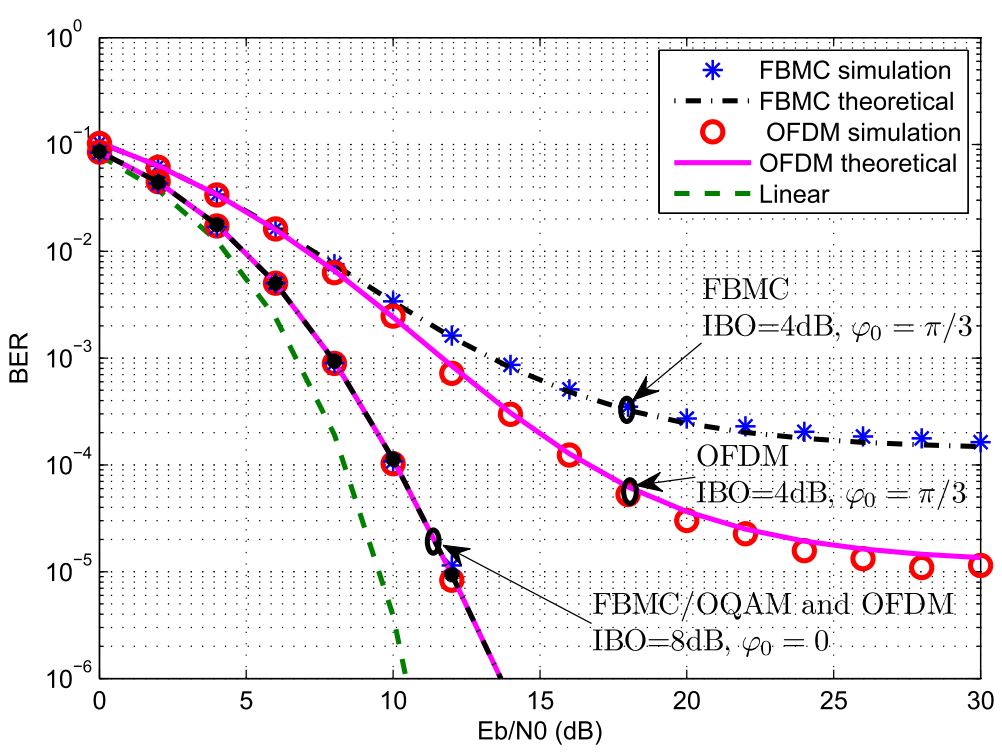

Figure 7 OFDM and FBMC/OQAM performance comparison. TWTA, 64 subcarriers, and AWGN channel. 


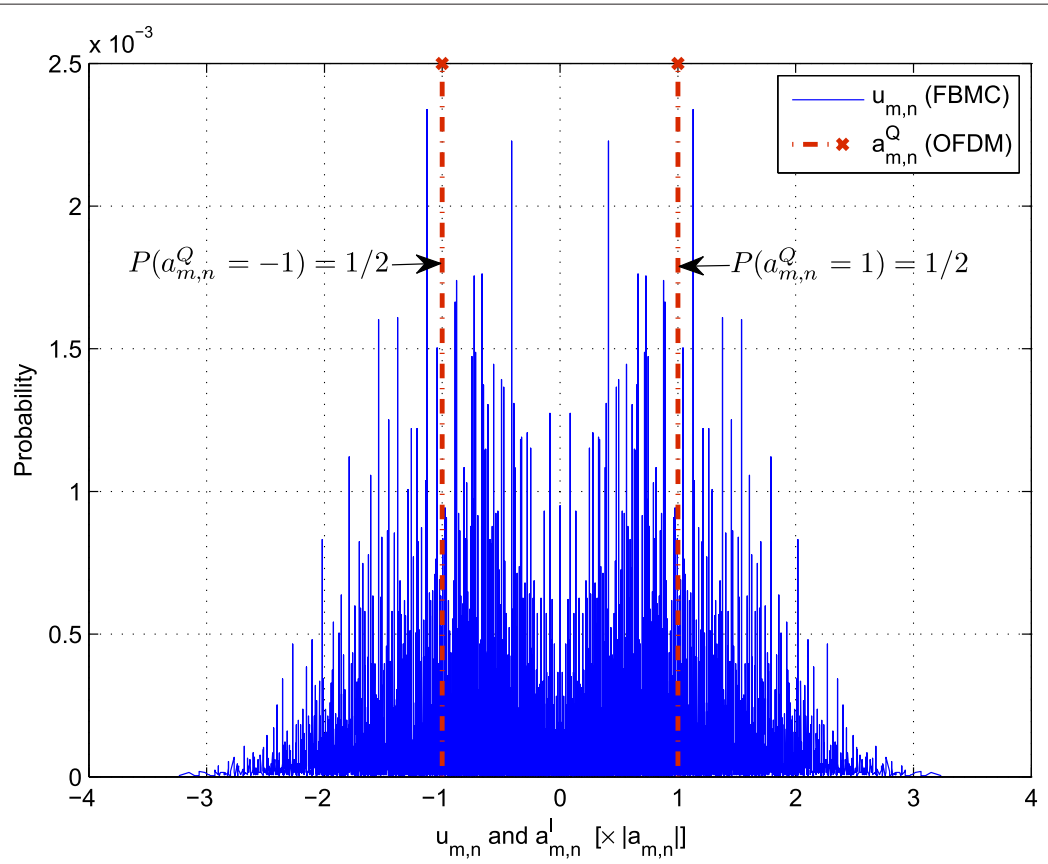

Figure 8 The respective distributions of $u_{m, n}$ and $a_{m, n}^{Q}$.

compensation for the NLD parameter $K$. In Figure 9, we present the results obtained in the presence of the SEL amplifier for both OFDM and FBMC/OQAM systems. From this figure, we can notice that both OFDM and FBMC/OQAM show the same performance. Additionally, there is an excellent match between the theoretical results and those obtained by the simulation.

In Figures 10 and 11, the performance of OFDM and FBMC/OQAM systems in the presence of TWTA with both $\mathrm{AM} / \mathrm{AM}$ and $\mathrm{AM} / \mathrm{PM}$ distortions is plotted. In these results, we compensate for the estimated NLD parameter $K$ at the receiver side. Figure 10 shows the OFDM and FBMC/OQAM performance in the case where only an AM/AM distortion is induced by the HPA. In Figure 11, a TWT HPA with $A_{\text {sat }}=1$ and $\varphi_{0}=\pi / 6$ is used. For both figures, the theoretical results are obtained by polynomial approximation of order 10 for the TWTA model. When there is only amplitude distortion, the case of Figure 10, both modulation schemes show the same performances which is also in accordance with the results

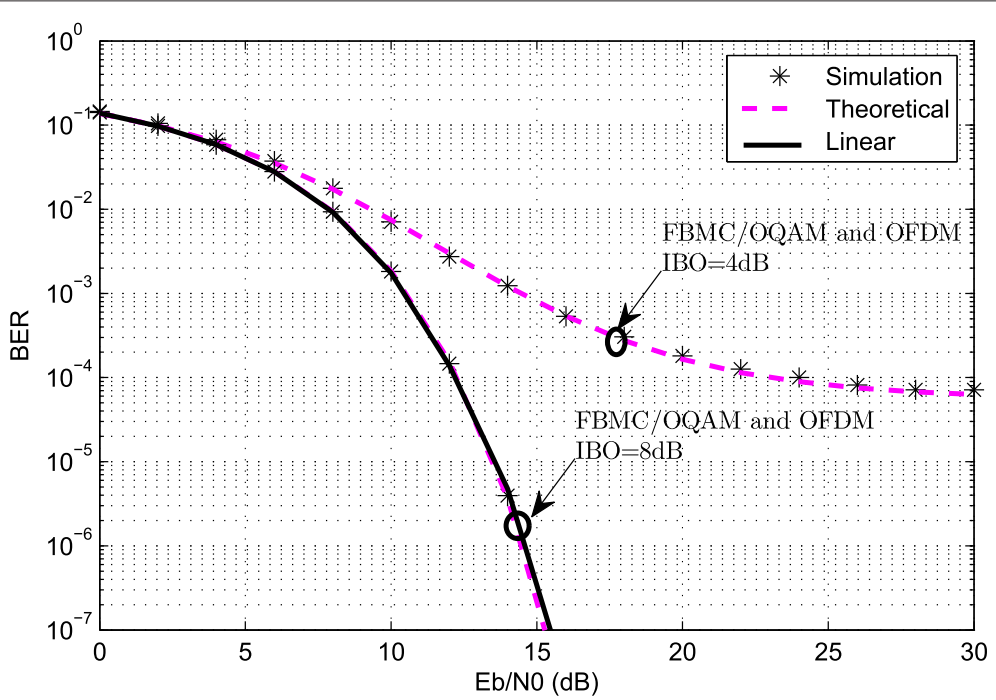

Figure 9 BER vs Eb/N0 for OFDM and FBMC/OQAM system. SEL, 64 subcarriers, 16QAM, and AWGN channel. 


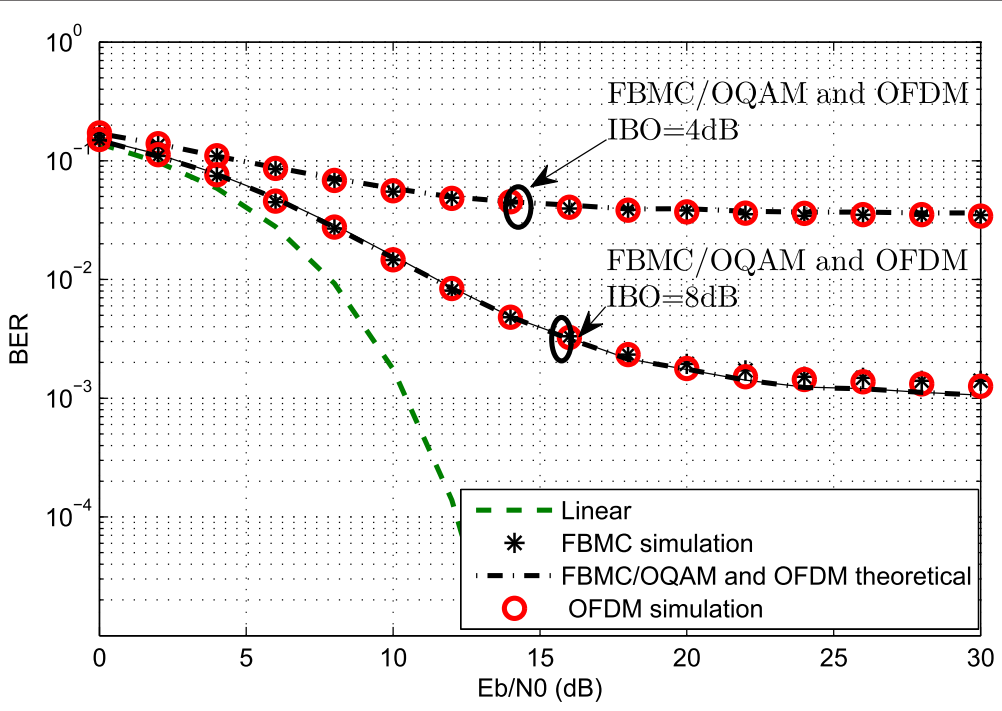

Figure 10 BER vs Eb/N0 for OFDM and FBMC/OQAM system. TWTA, 64 subcarriers, 16QAM, $\varphi_{0}=0$, and AWGN channel.

shown in Figure 9. When both amplitude and phase distortion are introduced by the HPA, the case of Figure 11 and after correction for the phase error, both OFDM and FBMC/OQAM modulations show the same performance. We confirm by this result that a perfect compensation for the parameter $K$ at the receiver side makes the FBMC/OQAM system as efficient as the OFDM one in terms of BER. This is the case in practice since the phase rotation induced by the NL parameter $K$ is taken into account by dedicated channel estimation and equalization algorithms.

\subsection{BER analysis in the case of a Rayleigh channel}

To further illustrate the effect of nonlinearity in the case of Rayleigh channel, the BER performance of
FBMC/OQAM, taking the 16QAM modulation scheme and TWTA with an IBO of $6 \mathrm{~dB}$, is shown in Figure 12. The Rayleigh channel was assumed to be a slowly varying flat fading one at a rate slower than the symbol duration $T$.

Based on Equation 41 and simulation results shown in Figure 12, we note that the BER, for relatively low $E_{b} / N_{0}$ (i.e., $E_{b} / \sigma_{w}^{2}<2 \mathrm{~dB}$ ), is very close to the BER performance of the Rayleigh channel with a linear HPA. This phenomenon is due to the fact that the residual degradation of the BER, which is caused by the nonlinear HPA, is negligible compared to the AWGN interference (i.e., $\sigma_{d} \ll \sigma_{w}$ ). Indeed, the SNR, given by Equation 52, has the same distribution as the Rayleigh one. At high values of $E_{b} / N_{0}$ (i.e., $E_{b} / \sigma_{w}^{2}>30 \mathrm{~dB}$ ), $\sigma_{w}$ is negligible and the

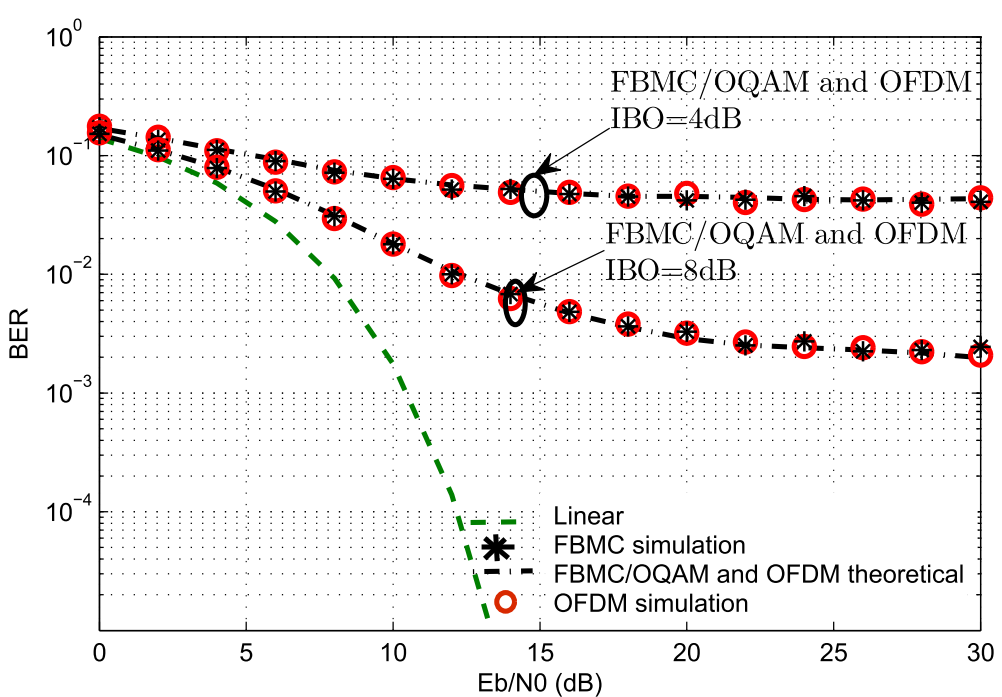

Figure 11 BER vs $E b / N 0$ for OFDM and FBMC/OQAM system. TWTA, 64 subcarriers, 16QAM, $\varphi_{0}=\pi / 6$, and AWGN channel. 


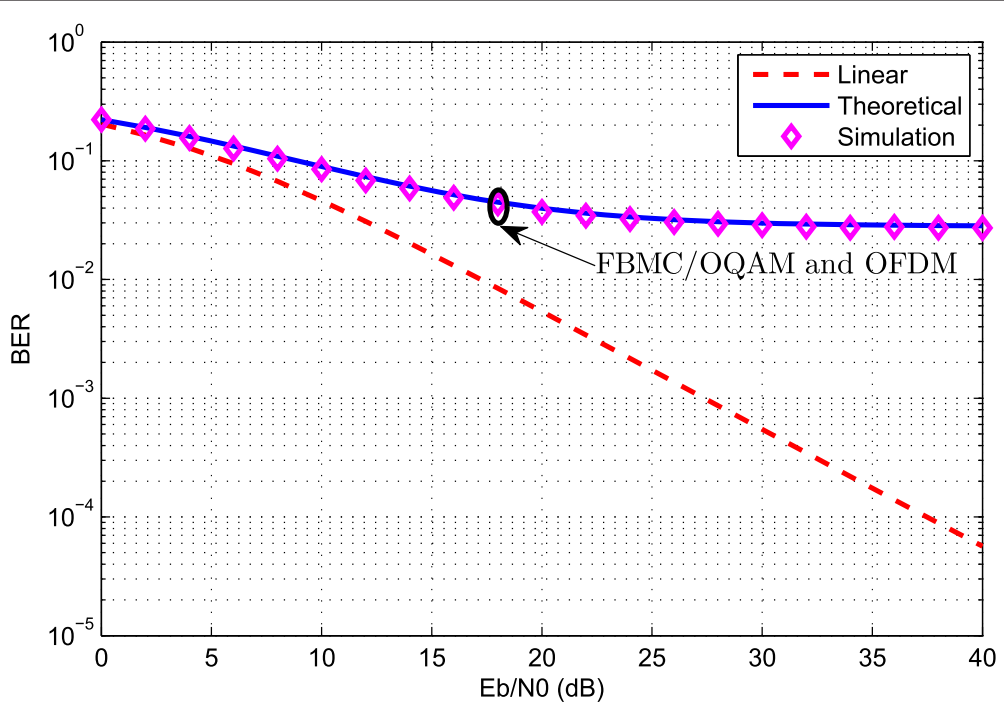

Figure $12 \mathrm{BER}$ vs $E b / N 0$ for OFDM and FBMC/OQAM system. TWTA, 64 subcarriers, $16 \mathrm{QAM}, \varphi_{0}=\pi / 3, \mathrm{IBO}=6 \mathrm{~dB}$, and Rayleigh channel.

SNR tends to a constant $\left(\gamma \rightarrow \frac{E_{b}}{\sigma_{d}^{2}}\right)$, leading then to a constant BER, as shown by Figure 12 .

\section{Conclusion}

In this paper, we have studied the impact of in-band nonlinear distortions caused by memoryless HPA on both OFDM and FBMC/OQAM systems. This study is valid for any measured or modeled HPA, exhibiting amplitude distortion (AM/AM) and phase distortion (AM/PM) over the multicarrier modulated signal. A theoretical approach was proposed to evaluate the BER performance for both OFDM and FBMC/OQAM systems. This approach is based on modeling the in-band nonlinear distortion with a complex gain and an uncorrelated additive white Gaussian noise, given by Bussgang's theorem. The theoretical determination of the NLD parameters is related to the HPA model used. For simple HPA models, the analytical expressions for the NLD parameters can be easily established. However, for more complicated HPA models exhibiting amplitude and phase distortions, the task is more complicated. The idea proposed in this paper is based on polynomial approximation of any HPA model at sufficient order. This makes possible the theoretical analysis for any measured or modeled HPA.

When only the amplitude of the modulated signals is distorted by the HPA, OFDM and FBMC/OQAM show the same performances in terms of BER. Simulations and theoretical results are shown to be in agreement for various IBO values. However, the FBMC/OQAM system is shown to be more sensitive to phase distortions than the OFDM one. The sensitivity of the FBMC/OQAM system to phase distortion is directly related to the intrinsic interference term introduced by this modulation.
This sensitivity cannot be seen as a limitation to FBMC modulation, since the phase error is practically taken into account during the channel estimation/equalization process.

\section{Competing interests}

The authors declare that they have no competing interests.

\section{Acknowledgements}

This work is supported by the European project EMPhAtiC (ICT- 318362) and the PHC-Utique C3 project (code 12G1414).

\section{Author details}

${ }^{1}$ CNAM - CEDRIC/LAETITIA, 292 rue Saint-Martin, Paris 75003, France. ${ }^{2}$ SUP'COM - INNOV'COM - Carthage University, Route de Raoued, Ariana 2083, Tunisia. ${ }^{3}$ ICTEAM, Université catholique de Louvain, Place du Levant, 2, Louvain-la-Neuve 1348, Belgium.

Received: 29 November 2013 Accepted: 17 April 2014 Published: 5 May 2014

\section{References}

1. BF Boroujeny, OFDM versus filter bank multicarrier. IEEE Signal Process. Mag. 28, 92-112 (2011)

2. G Wunder, M Kasparick, S ten Brink, F Schaich, T Wild, I Gaspar, E Ohlmer, S Krone, N Michailow, A Navarro, G Fettweis, D Ktenas, V Berg, M Dryjanski, S Pietrzyk, B Eged, [5GNOW]: challenging the LTE design paradigms of orthogonality and synchronicity, in IEEE VTC Spring (Dresden, Germany, 2013)

3. Y Dandach, P Siohan, Design method of OFDM/OQAM systems using a weighted time frequency localization criterion, in 18th European Signal Processing Conference, EUSIPCO (Aalborg, Denmark, 2010)

4. P Siohan, C Siclet, N Lacaille, Analysis and design of OFDM/OQAM systems based on filter bank theory. IEEE Trans. Signal Process. $\mathbf{5 0}$ 1170-1183 (2002)

5. MG Bellanger, Specification and design of prototype filter for filter bank based multicarrier transmission, in IEEE International Confrence on Acoustic, Speech and Signal Processing (Salt Lake City, USA, 2001), pp. 2417-2420

6. Y Medjahdi, M Terré, DL Ruyet, D Roviras, A Dziri, Performance analysis in the downlink of asynchronous OFDM/FBMC based multi-cellular networks. IEEE Trans. Wireless Comm. 10(8), 2630-2639 (2011)

7. B Imre, V Van, Central limit theorems for Gaussian polytopes. Ann. Probab. 35, 1593-1621 (2007) 
8. MI Abdullah, MZ Mahmud, MS Hossain, MN Islam, Comparative study of PAPR reduction techniques in OFDM. ARPN J. Syst. Software. 1, 263-269 (2011)

9. Z Kolar, P Horvath, Physical layer consideration for radio cognitive: modulation technique, in IEEE VTC Spring (Budapest, Hungary, 2011)

10. A Skrzypczak, P Siohan, J Javaudin, Analysis of the peak-to-average power ratio for OFDM/OQAM, in IEEE SPAWC (Cannes, France, 2006)

11. D Dardari, VTralli, A Vaccari, A theoretical characterization of nonlinear distortion effects in OFDM systems. IEEE Trans. Comm. 48(10), 1755-1764 (2000)

12. J Qi, S Aissa, On the effect of power amplifier nonlinearity on MIMO transmit diversity systems, in IEEE ICC (Dresden, Germany, 2009), pp. 2891-2895

13. J Qi, S Aissa, Analysis and compensation of power amplifier nonlinearity in MIMO transmit diversity systems. IEEE Trans. Vehicular Technol. 59, 2921-2931 (2010)

14. GT Zhou, Predicting spectral regrowth of nonlinear power amplifiers. IEEE Trans. Commun. 50, 718-722 (2002)

15. M Khodjet, C Saber, D Roviras, Y Medjahdi, Multi-carrier interference evaluation with jointly non-linear amplification and timing error, in IEEE VTC Spring (Budapest, Hungary, 2011)

16. H Bolcskei, Orthogonal Frequency Division Multiplexing Based on Offset QAM (Advances in Gabor Analysis, 2003), pp. 321-352

17. HE Rowe, Memoryless nonlinearity with gaussian inputs: elementary results. BELL Syst. Tech. J. 61(7), 1519-1526 (1982)

18. C Rapp, Effects of HPA nonlinearity on 4-DPSK-OFDM signal for digital sound broadcasting systems, in Second European Conf. on Sat. Comm (Liège, Belgium, 1991)

19. A Saleh, Frequency-independent and frequency-dependent nonlinear models of TWT amplifiers. IEEE Trans. Commun. 29, 1715-1720 (1981)

20. G Santella, F Mazzenga, A hybrid analytical-simulation procedure for performance evaluation in M-QAM-OFDM schemes in presence of nonlinear distortions. IEEE Trans. Vehicular Technol. 47, 142-151 (1998)

21. R Zayani, R Bouallegue, D Roviras, Adaptative pre-distortions based on neural networks associated with Levenberg-Marquardt algorithm for satellite down links. EURASIP J. Wireless Commun. Netw, 15 (2008). doi:10.1155/2008/132729

22. P Colantonio, F Giannini, E Limiti, High Efficiency RF and Microwave Solid State Power Amplifiers (Wiley, Hoboken, 2009)

23. SC Thompson, JG Proakis, JR Zeidle, The effectiveness of signal clipping for PAPR and total degradation reduction in OFDM systems, in IEEE GLOBCOM (St. Louis USA, 2005)

24. A Papoulis, Probability, Random Variables, and Stochastic Process, 3rd ed. (New York Polytechnic Institute, New York, 1991)

25. JJ Bussgang, Crosscorrelation Functions of Amplitude-distorted Gaussian Signals (1952). Research Laboratory of Electronics, Massachusetts Institute of Technology, Cambridge. Technical Report 216 (1952)

26. SC Cripps, RF Power Amplifier for Wireless Communications (Artech House, Norwood, 1999)

27. R Raich, H Qian, GT Zhou, Orthogonal polynomials for power amplifier modeling and predistorter design. IEEE Trans. Vehicular Technol. 53, 1468-1479 (2004)

28. JG Proakis, Digital Communications, 4th ed. (McGrawHill, New York, 2001)

29. A Leon-Garcia, Probability and Random Processes for Electrical Engineering (Addison-Welsey, Boston, 1994)

doi:10.1186/1687-6180-2014-60

Cite this article as: Bouhadda et al:: Theoretical analysis of BER performance of nonlinearly amplified FBMC/OQAM and OFDM signals. EURASIP Journal on Advances in Signal Processing 2014 2014:60.

\section{Submit your manuscript to a SpringerOpen ${ }^{\circ}$ journal and benefit from:}

- Convenient online submission

- Rigorous peer review

- Immediate publication on acceptance

- Open access: articles freely available online

- High visibility within the field

- Retaining the copyright to your article

Submit your next manuscript at $\boldsymbol{\nabla}$ springeropen.com 